\title{
Design and Development of an Industrial Network Laboratory
}

\author{
doi:10.3991/ijet.v6iS1.1615 \\ C.P. Leão, F.O. Soares, J.M. Machado, E. Seabra, H. Rodrigues \\ School of Engineering of Minho University, Guimarães, Portugal
}

\begin{abstract}
This paper presents the first developments of an industrial network laboratory prototype. In the Automation Laboratory several kits have been implemented for local and remote control tests. The approach includes the design and implementation of an industrial network where the common communications protocols can be tested: the Automation Network Locker (ANL). To facilitate the monitoring and control of the network, WALC (Web Assisted Laboratory for Control Engineering on-line Education) interface platform was developed. This allows the remote monitoring and control of the pedagogic kits to complement the teaching/learning automation to undergraduate engineering students. The security of network access is also taken into account in order to limit and control the number of users that can access it. An on-line questionnaire accessed at the end of each semester, allows an actualization and to follow the students' feedback.
\end{abstract}

Index Terms-Control Engineering, e-lab, Industrial Automation Systems, PLC network, Remote access.

\section{INTRODUCTION}

Remote laboratories and virtual animations are examples of interactive case-studies to be developed and used as learning environments in Process Control and Automation.

A simple search based on one or more keywords as "Virtual", "Remote", "Control", independent of the search tool used, gives, approximately, more than twenty thousand web pages that contains those words. This gives an idea of the multiple applications and the growing use of these resources in the Control teaching/learning: from Chemical Engineering to Medicine areas of applications [1-4]. In most of these works, the authors demonstrate the importance and the interest shown by students using these tools in their Control learning process [5].

In fact, the teaching/learning process has been under a detailed analysis, and severe changes have been slowly implemented. Above all, the attitude towards the education changed. The process is now centralized in the student. This implies not only a precise definition of objectives and capabilities to be acquired, but also strategies and teaching methodologies reformulation. The student is the main actor in the learning/teaching arena; he/she should be engaged in doing something besides listening to a lecturer and taking notes.

It is well known that students retain better what is taught if they are able to practice [6]. It comes into sight the concept "Learning by doing", particularly important for engineering students. Laboratories are then of utmost importance. Nevertheless, sometimes, it is difficult to have and maintain several working places as the devices are expensive, there is not enough laboratorial space and qualified personnel are not sufficient. With the use of the Internet as a vehicle of knowledge transmission and the availability of the wireless in the University campus, the Web laboratories can fill this gap, by optimizing resources, space and time.

So, executing laboratory experiments via the Internet, with the possibility of being performed anytime, anywhere, is of great interest for the emerging e-learning scenarios. The student can also have an immediate feedback, allowing him/her to work at his/her own pace, providing the opportunity to practice when and where he/she pleases.

In Engineering courses there are several Web Laboratories available: some works stand in a virtual environment with simulators, animations and videos [7]-[15] and are centered on electronics problems, process control and biomedical systems. Simulators are a compromise between dismissing hardware expenses of real experiences and a virtual perception of the problem. Nevertheless, we think that a clear observation of the real system is still needed. Several remote laboratories are available in the Internet [16]-[21] with applications, again, in electronics problems and process control but there is still a gap in what concerns automation subject. For example, there are not Programmable Logical Controllers (PLC) remotely available for students to test their real-world control programs, implementing different Ladder diagrams.

This paper describes the developments made by a multidisciplinary team of university investigators, from different backgrounds of engineering (mechanical, electronics, information systems and production and systems), and an industrial company of automation equipments and components, in the implementation of a web laboratory with remote control of experiments using diverse communications tools.

In order to achieve the main goals proposed on this paper, the paper is organized as follows: Section 1 presents the challenge of the work. In sections 2 and 3 it is presented a Description and Design of the ANL and the server communication used. Further, in section 4, it is presented the Educational Goal of the project. Finally, in section 5 there are listed the main conclusions of this study and some guidelines for the future work.

\section{DESCRIPTION AND DESIGN OF THE ANL}

In order to fulfill the main objective of implementing an industrial controllers' network, it is firstly necessary to define and to design the network functionality. This network integrates several prototypes that will be tested and includes also scalable models, such as car parks, 'home 
automation' kits, greenhouses, among others. The platform will allow not only the local communication and the connection of multiple devices to control and monitoring the prototypes but also it will allow the remote monitoring and control of the network.

A locker or electrical switchboard was designed to be used as the core of the network process control: Automation Network Locker (ANL) (Fig. 1). This locker contains a set of Programmable Logic Controller (PLC) that can be accessed through various communications tools: RS232, RS485, Mechatrolink, Profibus, DeviceNet and via Ethernet [22, 23]. The communication via Ethernet is considered the most important network communication since it is through this that the access to the PLCs programming and to the network control and to the several equipment's connections is performed [24, 25].

In Fig. 2 the ANL network is illustrated where different communication protocols are implemented, signaled by different colors.

The green line represents the DeviceNet communication and corresponding functions: exchange of data between control devices (Programmable Logic Controller, PLC), pro-visions of security devices and control inputs/outputs. The purple line represents the RS485 communication, containing two PLCs with different sensors. The orange is representing Mechatrolink communication to control the servomotor widely used for the transmission of power units. The Profibus, in blue/green line, links the

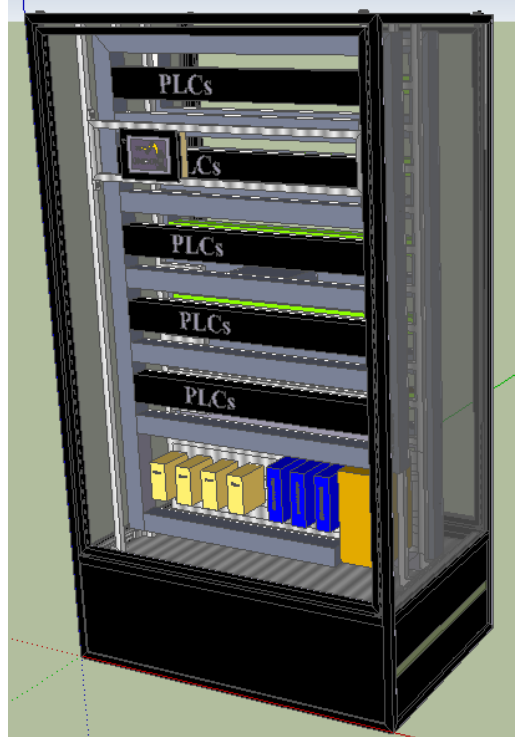

Figure 1. Automation Network Locker (ANL) prototype

inverter and a control device inputs/outputs. And finally the Ethernet network, blue line, contains the ANL network attached to two PLCs, a control device inputs/outputs and a vision system. It is the Ethernet network that takes the lead role of the network, since it allows the user (student) to remotely access between the networks.

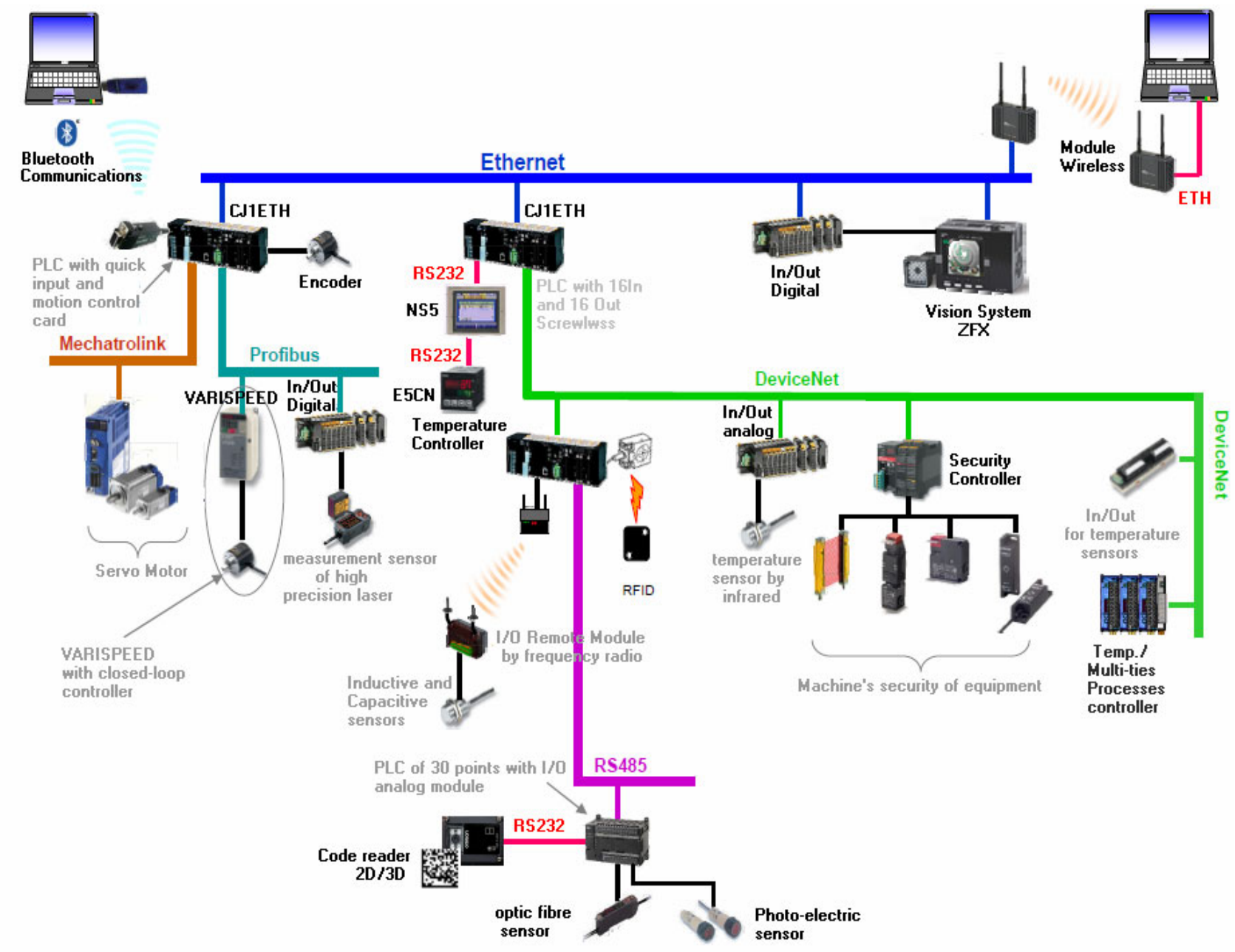

Figure 2. ANL network communications implemented 


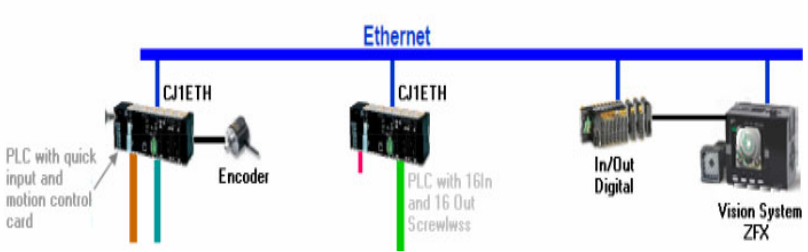

Figure 3. PLCs connected by Ethernet communication

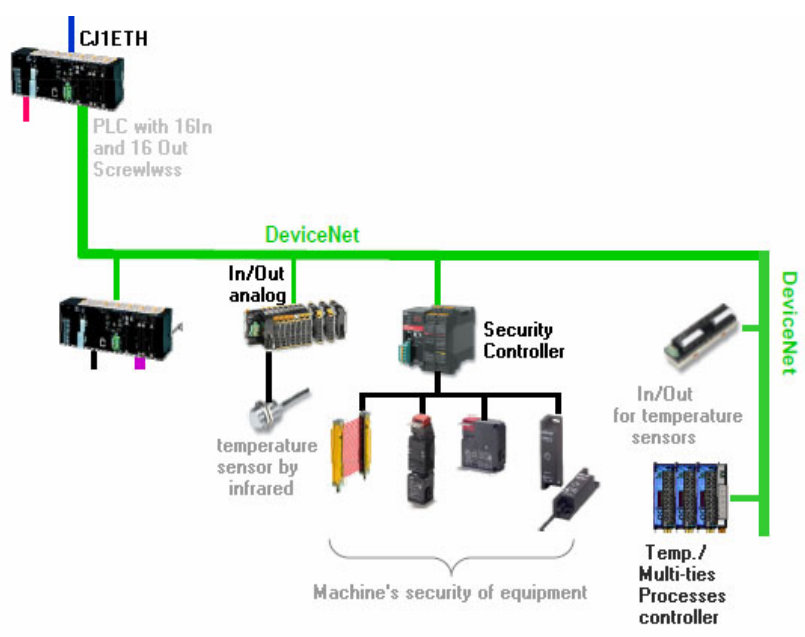

Figure 4. DeviceNet network

After the definition of the ANL network, each communication points between PLCs with IP address, the network is ready to be used either locally or remotely, and prepared to receive and send information from different PLCs in this network. The different equipment that constitutes the network, Ethernet, DeviceNet, Mechatrolink, Profibus, RS485 and RS232, will be described.

\section{A. Ethernet}

Ethernet is a technology for interconnecting local area networks. Local Area Networks (LAN) that is based on sending packets. Ethernet communication is linked to three of the four automata (Fig. 3).

\section{B. DeviceNet network}

The DeviceNet network is represented in Fig. 4, where the fourth PLC is integrated (considered as the most important PLC in this network) and connected to the second controller described in the network. As already mentioned, this communication is composed by the equipment used to control security devices [26].

\section{Mechatrolink}

Mechatrolink is a network system of industrial communication for the control of motion. Mechatrolink-has a transmission speed of $10 \mathrm{Mbps}$.

The communication control is established in the first PLC in Fig. 5, using the module CJ1M-MCH72 connected to the servomotor.

\section{Profibus network}

Profibus is the communication protocol extensively used in the market for the automation industry. Profibus is

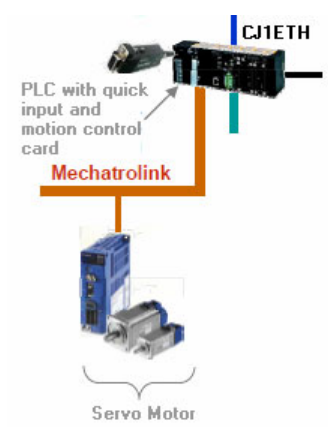

Figure 5. Mechatrolink network

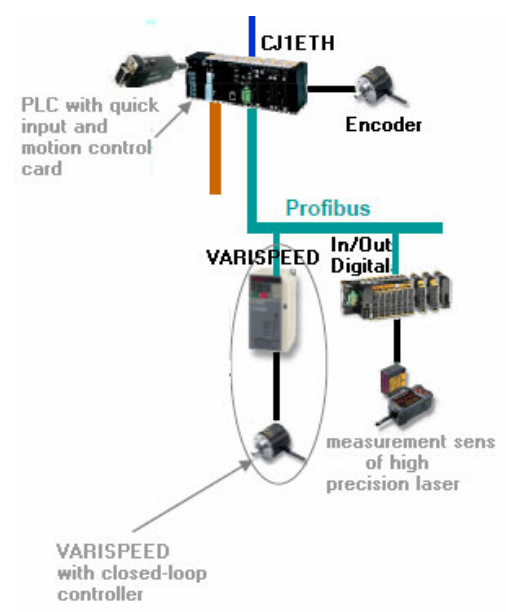

Figure 6. Profibus network

a standard open field network and independent, where the interface between them allows a wide application. Actually, the users can submit to an international standard protocol, which seeks to reduce development costs, flexibility, and diverse applications.

In the ANL network, the Profibus is connected to PLC 1 , which has a master module. Connected to the module is a control device inputs / outputs and an inverter (Fig. 6).

\section{E. RS485 network}

The RS485 network, also known as EIA485, is a communication protocol widely used in electronic devices. The data sending on the RS485 network is based on sending packets that switch between the "Master" and "Slaves". An RS485 network can be up to 32 knots, 12 meters supports communication at a maximum of $10 \mathrm{M}$ bps and the limit of distance supports a statement of $100 \mathrm{Kbps}(100 \times 1000$ bits per second $)$ at a distance of 1333 meters.

This network is very similar to the Profibus regarding devices connection. But while the Profibus protocol allows synchronization between manufacturers and it is possible to communicate with devices within different manufacturers, in RS485 communication it is difficult to connect devices from different manufacturers, due to the lack of RS485 communication protocol.

The RS485 network is connected to the PLC 4 (Fig. 7), consisting of a PLC with 30 points and display module $\mathrm{I} / \mathrm{O}$ analog and various sensors (optic fiber, photoelectric, among others). 


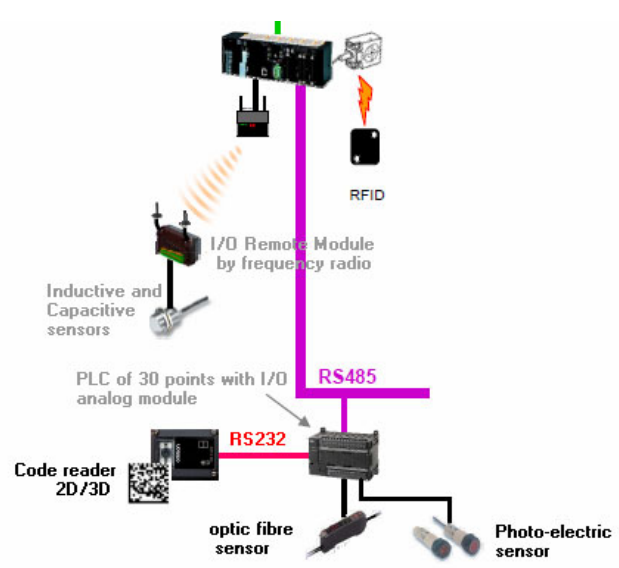

Figure 7. RS485 network

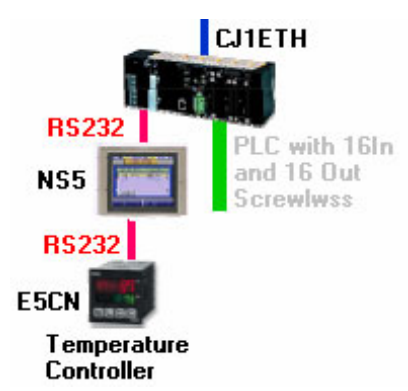

Figure 8. RS232 network

\section{F. RS232 network}

The network RS232, also known as EIA232, is a protocol where characters are sent in asynchronous mode, bit by bit, being necessary to send 10 bits to send a character.

This protocol contains an RS232 input, almost for connection to a computer for programming the equipment. The RS232 communication network used contains a touch screen console and a controlled temperature packer which in turn will be connected to PLC 2 (Fig. 8).

\section{G. Network control and monitoring}

The control and monitoring of networks have been growing due to the importance of this industry in the world. The management of monitoring and control of a network allows data acquisition in real time, the traffic characteristics, the diagnosis of faults and network configuration. Monitoring provides this information playing an important role in network management.

To monitor and control the ANL network, the Host Link and FINS protocols were used. These protocols give the necessary tool to get an effective network monitoring and control.

The monitoring and control centralization in a single point has been the main reason for the importance of innovation of PLC network, allowing a better management of the network. The development of a program for the network management gives to the user greater efficiency production.

To build this program was used the graphical programming language LabView ${ }^{\circledR}$. A prototype was developed in LabView with the ability to monitoring one or more PLCs (Fig. 9). With this prototype the user is able to select be-

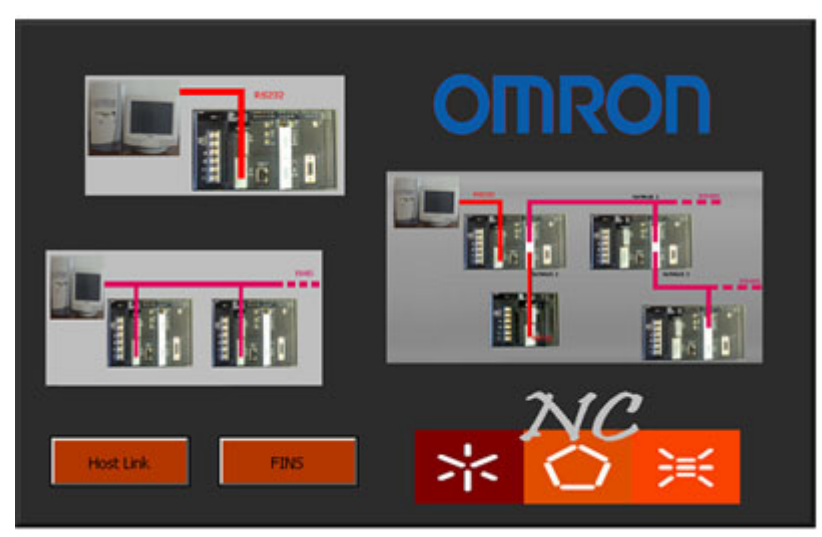

Figure 9. Interface front panel

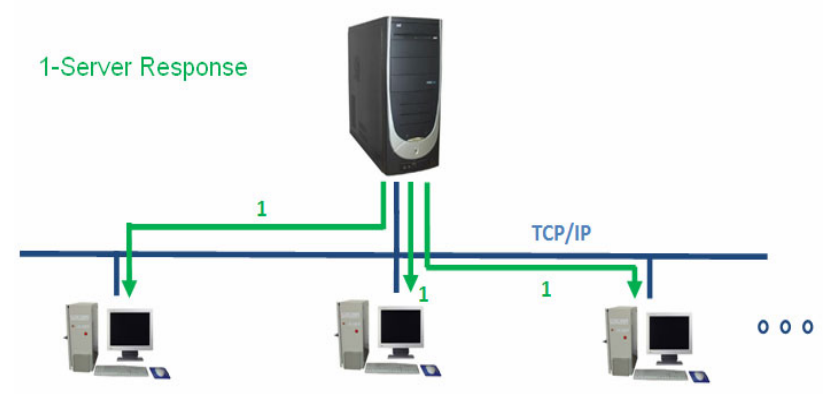

Figure 10. Server response

tween the two types of protocols available: Host Link and FINS (Factory Interface Network Service) protocols [27].

\section{Client/SERver COMMUNiCAtion}

The TCP/IP protocol is used on the Internet for sending and receiving user/student information when the access is made remotely. This system has been designed to accept several connected clients at the same time. Several difficulties in the communication emerge, especially in the recognition of data sent between the various clients and the server. The server response is directed to all the clients, as shown in Fig. 10, it was necessary to create a frame aimed at controlling the data flow. When there is only one client to access the server, there is no problem in terms of communication because there is only one user on the system.

Before the communication to the server is established, the client/student must first identify himself/herself, by giving name, course and e-mail. The server program, developed in LabView, manages all these specifications. After receiving a request from a client, the server must reply. The server response is then sent to all users, but in the client software, a comparison is performed between the frame and the client identification. If the comparison is true, the client executes the rest of the program. Otherwise, the user does not receive the information from the server.

\section{EdUCATIONAL GoAlS}

The ANL where the controller devices, relays and power supplies are centralized in a common space and dedicated case-study kits is linked to each PLC for realworld control tests. Moreover, the ANL can be easily 
transported to the classroom or exhibition for local control demonstrations.

To facilitate the remote process, monitoring and control, an interface, WALC (Web Assisted Laboratory for Control Engineering on-line Education) platform, was developed. Several practical control engineering problems and the control type implemented were implemented and available (Fig. 11). The available real-world problems are a DC Motor Control, the Temperature Control of a Classroom, BakSim (Baker's yeast Simulation), LabSim (Simulation Laboratory), Bar Control [28]. The first two practical cases are remote accessed real-world experiences, where the user can test a digital controller algorithm in motor velocity and in room temperature control. Both interfaces were developed in LabView from National Instruments. The last three, are virtual experiences, developed in Java and in LabView, This platform had included a reserved pass to the laboratory, managing a queue access list. By doing this, the student is able to access the ANL anytime and anywhere, testing his/her control program in a real-world case-study.

To obtain the feedback from students on the case-study kits implemented in the remote laboratory, an on-line questionnaire was prepared and set available on-line allowing that, each end of semester, the teacher and student can evaluate the practice performed. The questionnaire allows to understand and to analyze the students' reaction to the use of this tool in their learning process in Automation and Control. It is divided into six main parts: (1) student characterization, (2) work environment identification (operating system and browsers identification), (3) didactics kits motivation, (4) didactics kits technical skills, (5) didactic kits soft skills and (6) students habits and attitude characterization during the project.

The developed Control experiences were introduced as a learning tool in the Digital Control course on the 3rd year of the Industrial Electronics Engineering undergraduate course and in the Process Control course on the 2nd Industrial Informatics undergraduate course.

It could be pointed out some interesting conclusions obtained from a one year experience carried out. Nevertheless some difficulties in communications were identified: $94.1 \%$ of students mentioned that it was the first time that they participated in this type of experience. The University is the place by excellence to access the platform during the lunch period $(12 \mathrm{H}-14 \mathrm{H})$, being the afternoon period $(14 \mathrm{H}-18 \mathrm{H})$ the most used. This gives to the teacher an idea of the study hours that students prefer. The students share the access between the University and home.

The students' degree of concordance concerning their motivation in using the didactics kits as an appropriate tool on their Control and Automation learning process was very positive and must be continued. To follow the students' opinion, it is authors' believe to carry on this methodology implementing new kits developed by students for students.

At this moment, the interface communication was tested in a laboratory environment to remote monitoring and control a "small intelligent house" (Fig. 12).

With this interface several functionalities of the "small intelligent house" could be monitored and controlled, namely the alarm control, the temperature control, the entrance door open and closed and the illumination control.

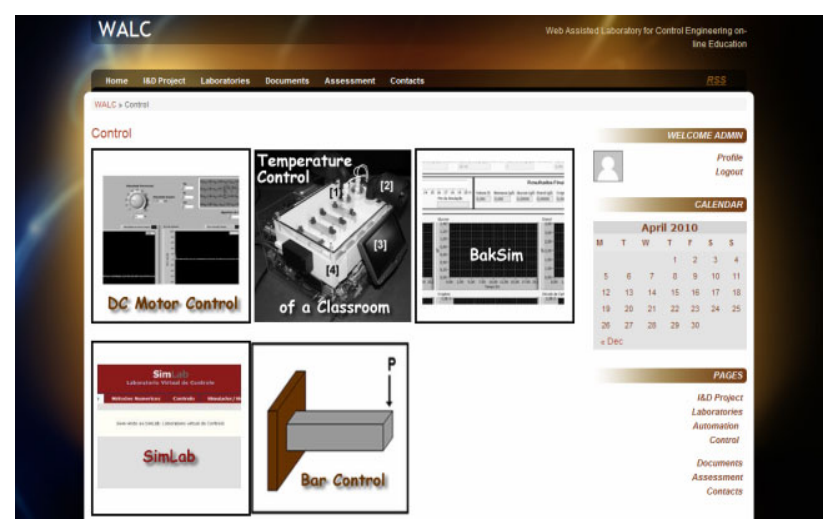

Figure 11. WALC platform interface for Control experiences

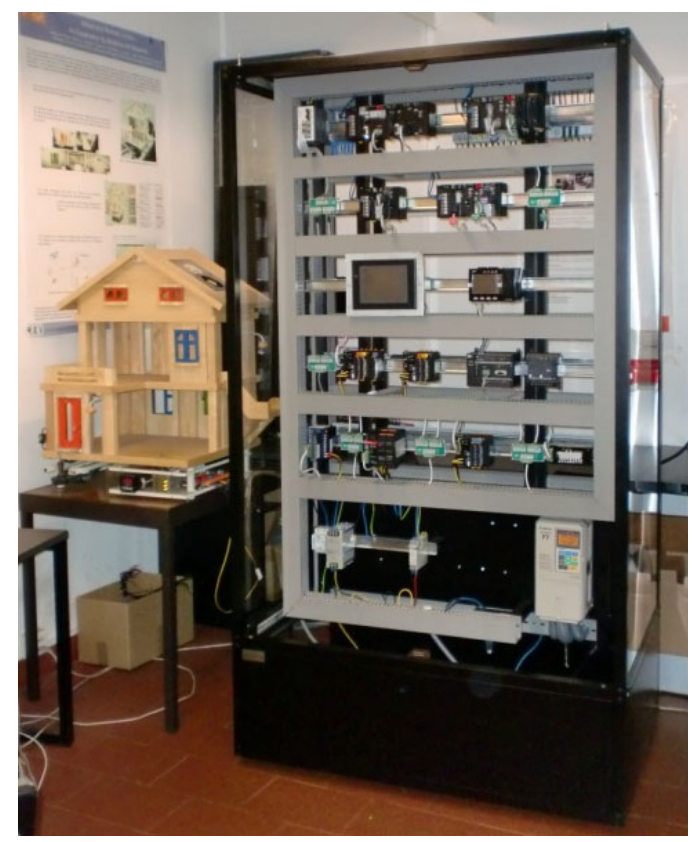

Figure 12. "small intelligent house" case-study for the ANL test

\section{FinAl COMmEnTS}

An ANL is being developed and implemented for teaching/learning automation and control subjects, either locally, in class, either remotely, through internet booked access. This last functionality will be implemented under WALC platform, the Automation Portal at Minho University. Among other benefits, the ANL allows the teaching of all Automation domains concerning all the Departments of School of Engineering of Minho University: this implies a rational use of human and physical resources.

The ANL includes the controller and the power supplies devices in a common closed space. Each real-world casestudy (mounted in a portable suitcase) is physical connected to the ANL for performing the tests.

It is the team' believe that with WALC, the user/student will be able to identify and follow their learning course in Automation and Control, by interacting with the interface parameters, testing system performance, defining inputs and analyzing results.

\section{ACKNOWLEDGMENT}

The authors are grateful to the students that participated in this project as designers and as end users. 


\section{REFERENCES}

[1] M. Stefanovic, V. Cvijetkovic, M Matijevic, V. Simic, “A LabVIEW-Based Remote Laboratory for Control Engineering Education", Comput Appl Eng Educ, 2009, Published on-line DOI doi:10.1002/cae.20334.

[2] B. Wagner, Professional Learning from Remote Sites, Proceedings of the International conference on Engineering Education, in ICEE 2007 Proceedings, Coimbra, Portugal, September 3-7, 4pp.

[3] V Pérez-Herranz, A.I Muñoz,.J.L Guiñón, J.Garcia-Antón, S.C Navarrete, An Internet-based Process Control Laboratory Project, Proceedings of the International Conference on Engineering Education, Valencia, Spain, 2003, July 21-25, 7 pp.

[4] S.D.Bencomo, "Control learning: present and future", Annual Reviews in Control, 28 (2004), 115-136. doi:10.1016/ j.arcontrol.2003.12.002

[5] M.Al-Khedher, D.B McDonald, Development of a Global WebBased Industrial Process Control Laboratory for Undergraduate and Graduate Engineering Technology Education, Proceedings of the 2008 IAJC-IJME International Conference, Nashville, USA, 2008, November 17-19, 10 pp.

[6] E.Hansen, "The role of interactive video technology in higher education: Case study and proposed framework", Education Technology, September 1990, 13-21.

[7] http://dei-s1.dei.uminho.pt/lic/AUT/ (accessed in December 2008).

[8] J. B. Olansen, E. Rosow, Virtual Bio-Instrumentation: Biomedical, Clinical, and Healthcare Applications in LabVIEW.

[9] http://www.imtc.gatech.edu/projects/education/bme.html (accessed in January 2009)

[10] http://www.clevemed.com/home/clevemed_education products.sh $\underline{\text { tml }}$ (accessed in January 2009)

[11] http://www.cs.cmu.edu/ dst/HHsim/ (accessed in January 2009)

[12] http://eidors3d.sourceforge.net/ (accessed in January 2009)

[13] http://www.pbs.org/wgbh/nova/heart/heartmap.html (accessed in January 2009)

[14] http://intro.bio.umb.edu/111112/112s99Lect/neuro_anims/s_t anim/WW36.htm (accessed in January 2009)

[15] http://www.nlm.nih.gov/research/visible/visible human.html (accessed in January 2009)

[16] G. Carneval, G.Buttazo, A Virtual Laboratory Environment for Real-time Experiments, Proceedings of the 5th IFAC International, Symposium on Intelligent Components and Instruments for Control Applications (SICICA 2003), Aveiro, Portugal, July 9-11, pp. 39-44, 2003.

[17] F. Coito, P. Almeida, L. B. Palma, SMCRVI-A Labview/Matlab Based Tool for Remote Monitoring and Control, 10 th IEEE Conference on Emerging Technologies and Factory Automation, ETFA 2005, Facolta' di Ingegneria, Catania, Italy, 19-22 September 2005 .

[18] http://isilab-esng.dibe.unige.it/ (accessed in January 2009)

[19] http://weblab2.mit.edu/docs/weblab/v6.1/manual/tutorial.htm (accessed in January 2009)

[20] http://www.apel.ee.upatras.gr/rmclab/en/index.htm (accessed in January 2009)
[21] Z. Nedic, J. Machotka, A. Nafalski, Remote Laboratories versus Virtual and Real Laboratories, 33rd ASEE/IEEE Frontiers in Education Conference, Boulder, CO, November 5-8, 2003

[22] D. M. Considine, Standard Handbook of Industrial Automation, Champman and Hall, UK, 1986.

[23] K. Lee, Sensor Networking and Interface Standardization, IEEE Instrumentation and Measurement Technology Conference, Budapest, Hungary, 2001, May 21-23, 6 pp.

[24] M. Felser, Real-time Ethernet - industry prospective, Proceedings of the IEEE, 93 (6), June 2005, pp. 1118 - 1129. doi:10.1109/ JPROC.2005.849720

[25] J.M. Kizza, Security Threats to Computer Networks, Computer Communications and Networks Part II, Springer, USA, 2005.

[26] DeviceNet Network, http://pt.wikipedia.org/wiki/ DeviceNet (accessed in October 2009)

[27] N.Carvalho, C.P.Leão, F.O. Soares, J. Machado, An Interface for industrial network monitoring and control, 10th IFAC Workshop on Intelligent Manufacturing Systems (IMS'10), Lisboa, Portugal, July 2010, 6pp.

[28] S. Costa, C.P. Leão, F. Soares, H. Rodrigues, J. Machado, WALC Web Assisted Laboratory for Control Engineering On-line Education, CONTROLO2010 Conference, Coimbra, Portugal, 8-10 September 2010 .

\section{AUTHORS}

C. P. Leão, is with the Production and Systems Engineering Department (Numerical Methods and Statistical Group), Engineering School of University of Minho, Campus of Gualtar, 4710-057 Braga, Portugal (e-mail: cpl@dps.uminho.pt).

F. O. Soares, is with the Industrial Electronics Department, Engineering School of University of Minho, Campus of Azurém, 4800-058Guimaraes, Portugal (e-mail: fsoares@dei.uminho.pt).

J. M. Machado, is with the Mechanical Engineering Department, Engineering School of University of Minho, Campus of Azurém, 4800-058Guimaraes, Portugal (email: jmachado@dem.uminho.pt).

E. Seabra, is with the Mechanical Engineering Department, Engineering School of University of Minho, Campus of Azurém, 4800-058Guimaraes, Portugal (email: eseabra@dem.uminho.pt).

H. Rodrigues, is with the Information System Department, Engineering School of University of Minho, Campus of Azurém, 4800-058Guimaraes, Portugal (e-mail: hcr@dsi.uminho.pt).

This work was supported in part by the Portuguese Foundation for Science and Technology (FCT) for funding through the R\&D project PTDC/ESC/68069/2006.

This article was modified from a presentation and paper at The 10th International Virtual University Conference, in 10-11 December 2009, Bratislava, Slovak Republic.

Received, March $25^{\text {th }}, 2011$. Published as resubmitted by the authors on April 20, 2011. 